\title{
Interactions and conformational fluctuations in DNA arrays
}

\section{Rudolf Podgornik}

Laboratory of Physical and Structural Biology

National Institute of Child Health and Human Development

National Institutes of Health

9 Memorial Drive, MSC 0924

Bldg. 9, Rm. 1E116

Bethesda, MD 20892-0924

\section{ABSTRACT}

I will give a broad overview of recent work on the equation of state of DNA in aqueous monovalent salt solutions. The picture that I will develop shows that at non-negligeable monovalent salt concentrations the direct electrostatic interactions between DNA molecules are almost always masked by the thermal conformational fluctuations of the DNA chains. These thermal fluctuations act to boost the magnitude as well as the spatial range of the electrostatic interactions. This renormalization of the bare electrostatic interactions is a salient feature of dense systems composed of flexible polyelectrolyte moleclues.

\section{INTRODUCTION}

In the phase diagram of DNA in aqueous solutions there is a range extending from the DNA crystal at densities corresponding to interaxial separations below $\approx 24$ all the way to the nematic - isotropic transition at densities corresponding to interaxial separations $\approx 120$ (for a bathing solution of $0.5 \mathrm{M} \mathrm{NaCl}$ ) where DNA is orientationally ordered or even shows long range hexatic order perpendicular to the long axes of the molecules but is positionally still a liquid with only short range positional order [1]. This is in particular valid for long $\sim \mu m$ fragments of DNA. The local structure of these DNA arrays is presented on Fig. 2. In this part of the phase diagram, the equation of state, i.e. the dependence of the osmotic pressure on the macromolecular (DNA) concentration, has been 
studied very carefully [2]. In this lecture I will give a comprehensive introduction to the equation of state for DNA under various solution conditions with monovalent salts and counterions and make an attempt to explain in some detail the theoretical underpinnings of its equation of state.

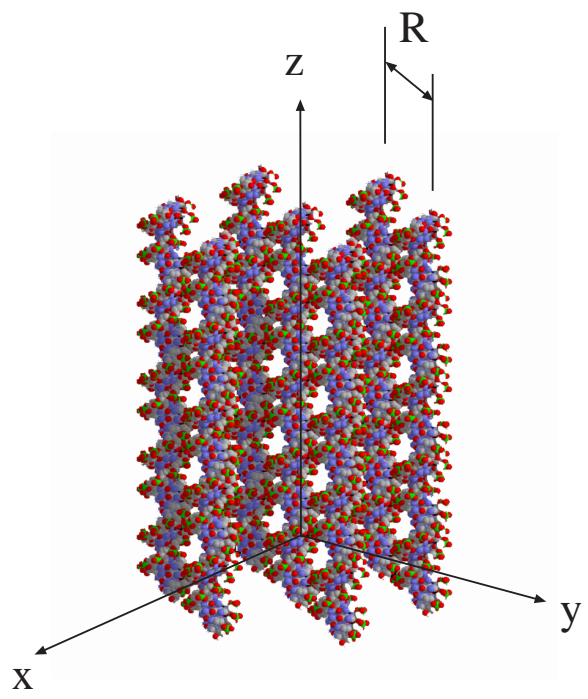

Figure 1. The schematic geometry of an array of DNA molecules with long range orientational order and short range positional order. Drawn approximately to scale. $R$ is the average interaxial separation between the molecules.

\section{ELECTROSTATIC INTERACTIONS}

Electrostatic interactions between charged macromolecules are one of the two pillars of the DLVO theory of colloid stability [3, 4]. The other one being the Lifshitz - van der Waals electromagnetic fluctuation forces [5]. In DLVO theory both contributions are assumed to be additive. In general however, the van der Waals forces and the electrostatic interactions are coupled through the s.c. zero-order term of the Lifshitz formula [5]. This coupling is important in cases where counterion correlations make a large attractive contribution to the total interaction as e.g. in the case of polyvalent counterions.

In what follows I will dwell exclusively on the electrostatic component of the DLVO theory, assuming that the van der Waals forces are negligeable in the context of DNA interactions, their magnitude being always much smaller then the magnitude of the electrostatic interactions. I will also not discuss the water-mediated structural interactions that make their mark on the equation of state at very high DNA densities and/or high salt concentrations. 


\subsection{Poisson-Boltzmann theory}

In our investigation of the electrostatic interactions between charged macromolecules we will start by writing down the expression for the non-equilibrium mean-field free energy density $(f)$ of a gas of mobile charged particles i.e. counterions and salt ions. It can be written as a difference of the electrostatic field energy density $(w)$ and the ideal entropy density of the mobile charge carriers $(s)$ [6]. The electrostatic field energy density can be written as

$$
w\left(\mathbf{E}(\mathbf{r}), \rho_{i}(\mathbf{r})\right)=-\frac{1}{2} \epsilon \epsilon_{0} \nabla \phi(\mathbf{r})^{2}(\mathbf{r})+\sum_{i} z_{i} e_{i} n_{i}(\mathbf{r}) \phi(\mathbf{r}),
$$

where $\mathbf{E}(\mathbf{r})=-\boldsymbol{\nabla} \phi(\mathbf{r})$ is the local electrostatic field and $\epsilon$ is the static dielectric constant and $n_{i}$ is the density of the charged species $i$, of charge $z_{i} e_{i}$. The total charge density is thus $\sum_{i} \rho_{i}(\mathbf{r})=\sum_{i} z_{i} e_{i} n_{i}(\mathbf{r})$. After minimizing this expression w.r.t. $\phi(\mathbf{r})$ one remains with the standard Poisson equation and the usual form of the electrostatic filed energy density.

Let us assume furthermore that there are $N$ mobile ionic species with charges $z_{i} e_{i}$, whose densities are $n_{i}$ while $n_{i}^{0}$ is the particle density of the same mobile charged species in the bulk with which the system is in chemical equilibrium. Then the entropy difference between the volume under consideration and the bulk is given by

$$
s\left(n_{i}(\mathbf{r})\right)=-k_{B} \sum_{i}\left(n_{i}(\mathbf{r}) \log \left(\frac{n_{i}(\mathbf{r})}{n_{i}^{0}}\right)-\left(n_{i}(\mathbf{r})-n_{i}^{0}\right)\right),
$$

where $k_{B}$ is the Boltzmann constant. The complete non-equilibrium free energy difference is then defined as a volume integral

$$
\mathcal{F}=\int d^{3} \mathbf{r} f\left(\mathbf{E}(\mathbf{r}), n_{i}(\mathbf{r})\right)=\int d^{3} \mathbf{r}\left(w\left(\mathbf{E}(\mathbf{r}), n_{i}(\mathbf{r})\right)-T s\left(n_{i}(\mathbf{r})\right)\right) .
$$

By minimizing the free energy Eq. 3 with respect to the electrostatic potential one first obtains the Euler - Lagrange equation in the form

$$
\boldsymbol{\nabla}\left(\frac{\partial f}{\partial \boldsymbol{\nabla} \phi}\right)+\frac{\partial f}{\partial \phi}=0 \quad \text { wherefrom } \quad \epsilon \epsilon_{0} \boldsymbol{\nabla} \mathbf{E}(\mathbf{r})=\sum_{i} z_{i} e_{i} \rho_{i}(\mathbf{r})
$$

This is obviously nothing but the Poisson equation. By furthermore minimizing the free energy Eq. 3 with respect to ionic densities $n_{i}$ one obtains the Euler - Lagrange equation in the form

$$
\frac{\partial f}{\partial n_{i}}=0 \quad \text { wherefrom } \quad z_{i} e_{i} \phi(\mathbf{r})+k_{B} T \log \left(\frac{n_{i}(\mathbf{r})}{n_{i}^{0}}\right)=0 .
$$

Taking into account the Poisson equation Eq. 4, one can rewrite the above equation as the Poisson - Boltzmann equation

$$
\nabla^{2} \phi(\mathbf{r})=-\frac{1}{\epsilon \epsilon_{0}} \sum_{i} \rho_{i}(\phi(\mathbf{r}))=-\frac{e_{0}}{\epsilon \epsilon_{0}} \sum_{i} z_{i} n_{i}^{0} e^{-\beta z_{i} e_{0} \phi(\mathbf{r})},
$$

that gives the equilibrium (mean-field) profile of the electric field in the system. If one integrates the Poisson equation over the whole volume available to the mobile ionic species 
and takes into account the theorem of Gauss- Ostrogradsky, one gets

$$
\epsilon \epsilon_{0} \oint_{\partial V}(\mathbf{E} \cdot \mathbf{n}) d^{2} \mathbf{r}=\int_{V} \sum_{i} e_{i} \rho_{i}(\mathbf{r}) d^{3} \mathbf{r}=\oint_{\partial V} \sigma d^{2} \mathbf{r} .
$$

Here a further assumption is that the system is overall electroneutral and thus the total volume charge should be matched by the neutralizing surface charge of surface charge density $\sigma$ on the boundaries of the volume. Further details on the derivation of the Poisson - Boltzmann (PB) equation can be found in [6],

If the magnitude of the surface charges is not too large, one has $\beta e_{0} \phi(\mathbf{r}) \ll 1$ in the whole accessible volume. Assume further the we have only two mobile charged species: $e_{1}=e_{0}$ and $e_{2}=-e_{0}$, together with $n_{1}=n_{+}$and $n_{2}=n_{-}$, in equilibrium with a bulk reservoir with $n_{i}^{0}=n_{0}$. In this case the Poisson-Boltzmann equation can be linearized and reduced to the Debye - Hückel equation [6]

$$
\nabla^{2} \phi(\mathbf{r})=-\frac{e_{0} n_{0}}{\epsilon \epsilon_{0}} \sum_{i} z_{i} e^{-\beta z_{i} e_{0} \phi(\mathbf{r})} \approx-\frac{e_{0} n_{0}}{\epsilon \epsilon_{0}} \sum_{i}\left(z_{i}-\beta\left(z_{i}\right)^{2} e_{0} \phi(\mathbf{r})+\ldots\right)=\kappa_{D}^{2} \phi(\mathbf{r}) .
$$

By assumption $\sum_{i} z_{i}=0$ and $\sum_{i}\left(z_{i}\right)^{2}=2$. Above we have introduced

$$
\kappa_{D}^{2}=8 \pi \ell_{B} n_{0} \quad \text { where } \quad \ell_{B}=e_{0}^{2} / 4 \pi \epsilon \epsilon_{0} k_{B} T
$$

is the Bjerrum length, defined to be the separation between two elementary charges at which their electrostatic interaction energy equals the thermal energy. At room temeperature and water as solvent it's value is 7.1. The constant $\kappa_{D}=\lambda_{D}^{-1}$ is interpreted as the inverse Debye screening length. For monovalent salts at room temperature with concentration expressed in moles $[\mathrm{M}]$ per liter, $\lambda_{D}=3.05 / \sqrt{[M]}$ in $\AA$. The salient feature of electrostatic interaction on the linearized PB equation level is the screening quantified by the Debye length $\lambda_{D}$, leading to an approximately exponential decay of the interactions as a function of the separation between the charges. This picture of screened interactions has to be drastically modified in the case of polyvalent counterions [7].

The linearization of the PB equation is usually justified at asymptotic conditions, meaning usually a small surface charge and/or a large separation between charged macroions as well as by the fact that it is easily amenable to analytic solutions. The full non-linear $\mathrm{PB}$ equation represents a much tougher mathematical problem analytically solvable only for special geometries.

\subsection{The cell model}

In many colloidal systems, most notably in the case of ordered DNA phases, one seldom deals with isolated molecules in ionic solutions. Quite often one has a phase of densely packed macroions which complicates the problem of evaluating the electrostatic interactions even further. A simple way around this problem is the polyelectrolyte cell model [8], being a variant of the Wigner - Seitz model of electron in the crystalline lattice, which substitutes the complicated colloidal geometry with a cell, containing a single colloid. The effect of the rest is assumed to be mimicked by the cell wall where the electrostatic potential should have a zero derivative by symmetry. 
For a long cylindrical molecule such as DNA in a dense phase where molecules are on the average oriented in one direction, a cylindrical cell model should capture the main features of the molecular environment. There are nevertheless important caveats that one has to be aware of in the context of the cell model [9]. The linearized PB equation in cylindrical coordinates $(r, \theta, z)$ reads

$$
\frac{1}{r} \frac{d}{d r}\left(r \frac{d \phi}{d r}\right)=\kappa_{D}^{2} \phi
$$

with the boundary condition at the inner wall, (i.e. at the surface of the central molecule with a radius $a$ ) being

$$
\frac{d \phi}{d r}(r=a)=-\frac{\sigma}{\epsilon \epsilon_{0}}=-\frac{e_{0}}{2 \pi \epsilon \epsilon_{0} a b},
$$

wher $b$ is the length of the molecule per one elementary charge on the surface. For DNA its structural charge would correspond to one charge per $1.7 \AA$. The boundary condition at the outer surface of the cell, assumed to be located at $r=R$, is by symmetry

$$
\frac{d \phi}{d r}(r=R)=0
$$

The radius of the cell $R$ is obtained from the macromolecular density of the system. If the density of the macromolecules is $n_{M}$, then $n_{M}{ }^{-1}=\pi\left(R^{2}-a^{2}\right) b$. The macromolecular density thus determines the radius of the cell.

Obtaining a solution of the linearized Poisson - Boltzmann equation in cylindrical cell model is quite straightforward and leads to the electrostatic potential that can be expressed via the cylindrical Bessel functions $K_{0}(x)$ and $I_{0}(x)$ as

$$
\phi(r)=\frac{e_{0}}{2 \pi \epsilon \epsilon_{0} b\left(\kappa_{D} a\right)} \frac{K_{0}\left(\kappa_{D} r\right) I_{1}\left(\kappa_{D} R\right)+I_{0}\left(\kappa_{D} r\right) K_{1}\left(\kappa_{D} R\right)}{K_{1}\left(\kappa_{D} a\right) I_{1}\left(\kappa_{D} R\right)-I_{1}\left(\kappa_{D} a\right) K_{1}\left(\kappa_{D} R\right)} .
$$

From here we get for the radial component of the electrostatic field the expression

$$
E_{r}(r)=-\frac{d \phi}{d r}=\frac{e_{0}}{2 \pi \epsilon \epsilon_{0} b a} \frac{K_{1}\left(\kappa_{D} r\right) I_{1}\left(\kappa_{D} R\right)-I_{1}\left(\kappa_{D} r\right) K_{1}\left(\kappa_{D} R\right)}{K_{1}\left(\kappa_{D} a\right) I_{1}\left(\kappa_{D} R\right)-I_{1}\left(\kappa_{D} a\right) K_{1}\left(\kappa_{D} R\right)} .
$$

One should note here that for a single cylinder, thus in the limit of $R \longrightarrow \infty$, the electrostatic potential and the electrostatic field reduce to

$\lim _{R \longrightarrow \infty} \phi(r)=\frac{e_{0}}{2 \pi \epsilon \epsilon_{0} b\left(\kappa_{D} a\right)} \frac{K_{0}\left(\kappa_{D} r\right)}{K_{1}\left(\kappa_{D} a\right)} \quad$ and $\quad \lim _{R \longrightarrow \infty} E_{r}(r)=\frac{e_{0}}{2 \pi \epsilon \epsilon_{0} b a} \frac{K_{1}\left(\kappa_{D} r\right)}{K_{1}\left(\kappa_{D} a\right)}$.

Additional terms in the cell model solution Eq. 13 and 14 are thus due to the finite concentration of the DNAs and obviously depend on its density via the radius of the outer cell wall $R$. One should note here that the linearized solutions of the PB equation are quite accurate in the case that the salt concentration is not too small [10]. The nonlinear PB equation has an analytical solution [11] only for a single cylinder in infinite ionic solution, 


\subsection{Osmotic pressure}

The forces between macromolecules mediated by the equilibrium distribution of counterions and salt ions between them can be obtained via the stress tensor at the outer surface of the cell, which gives the force acting on this surface. This force per unit area of the cell is obviously nothing but the osmotic pressure. The stress tensor contains the Maxwell electrostatic part and the osmotic part [12],

$$
\sigma_{i j}=\epsilon \epsilon_{0}\left(E_{i} E_{j}-\frac{1}{2} E^{2} \delta_{i j}\right)-k_{B} T \sum_{i} n_{i}(\phi(\mathbf{r})) \delta_{i j} .
$$

The last term is simply the van's Hoff ideal gas pressure, corresponding to the ideal gas entropy in the free energy ansatz. The negative sign comes from the general continuum mechanics argument that positive pressure should lead to a decrease in volume.

We can evaluate the stress tensor on any plane of cylindrical symmetry witin the cell. In many cases it is simplest to take the stress tensor at the surface of the central macromolecule, in which case the forces are obtained via a contact theorem [12]. Equivalently we can take the stress tensor at the outer wall of the cell where by symmetry the electric field is zero and thus the stress tensor contains only the van't Hoff part. Since the latter case is simpler we thus find for the radial components of the stress tensor (all the other ones vanish at the outer wall)

$$
\sigma_{r r}=-k_{B} T \sum_{i} n_{i}(r=R) .
$$

The osmotic pressure $\Pi(R)$ in the cell is via the mechanical equilibrium, equal to minus the force per surface area on the outer wall, thus

$$
\Pi(R)=-\sigma_{r r}=k_{B} T \sum_{i} n_{i}(r=R) .
$$

We can use this expression to derive the form of the osmotic pressure in the case of the linearized PB equation in a cylindrical cell. Remember that we have assumed that we have only two mobile charged species: $e_{1}=e_{0}$ and $e_{2}=-e_{0}$, together with $n_{1}=n_{+}$ and $n_{2}=n_{-}$, in equilibrium with a bulk reservoir where $n_{1}^{0}=n_{2}^{0}=n_{0}$. The osmotic pressure difference between the cell and the bulk reservoir, which alone is measurable, can be evaluated via the expression Eq. 18

$$
\begin{aligned}
\Pi & =k_{B} T\left(n_{+}(r=R)+n_{-}(r=R)\right)-2 k_{B} T n_{0}= \\
& =2 k_{B} T n_{0}\left(\cosh \beta e_{0} \phi(r=R)-1\right) .
\end{aligned}
$$

This is the complete expression for osmotic pressure difference between the inside of the cell and the bulk. Obviously here the only molecular species contributing to the osmotic pressure are the mobile ions. The macromolecule itself does not contribute to the osmotic pressure. We will see later that this point of view is not entirely correct.

Another approach to the evaluation of the osmotic pressure, independent of the cell model, would be to calculate the complete equilibrium free energy $\mathcal{F}$ from Eq. 3 via a volume integral over all the space available to the mobile charges

$$
\mathcal{F}\left[n_{M}\right]=\int d^{3} \mathbf{r} f\left(\mathbf{E}(\mathbf{r}), n_{i}(\mathbf{r})\right)
$$


where the electrostatic field and the densities of the mobile charges are obtained from the solution of the full or linearized Poisson - Boltzmann equation Eq. 6. This free energy is of course a function of the concentration of the macromolecules $n_{M}$. The osmotic pressure in the system would then be obtained from the standard thermodynamic relation

$$
\Pi=-\left(\frac{\partial \mathcal{F}\left(n_{M}\right)}{\partial V_{M}}\right)_{T, \mu} .
$$

Constant temperature and chemical potential of the mobile charged species are of course assumed in the above expression. In the case that the macromolecular solution is ordered and exhibits certain symmetries, this expression can be simplified even further. If we again assume that the effects of the packing symmetry of the molecules can be captured by a cylindrical cell model of radius $R$ and that the length of the molecules is $L$, we can write for the osmotic pressure

$$
\Pi=-\left(\frac{\partial \mathcal{F}\left(n_{M}\right)}{\partial V_{M}}\right)_{T, \mu}=-\left(\frac{\partial(\mathcal{F}(R) / L)}{2 \pi R \partial R}\right)_{T, \mu} .
$$

Eq. 22 connects the expression for the osmotic pressure in the cell model with the one obtained from the complete partition function. Discrepancies between the values for osmotic pressure obtained from the two expressions are due to the approximate nature of the cell model.

\subsection{Interaction between cylindrical macromolecules}

Let us now investigate the osmotic pressure in the cylindrical cell model on the level of the linearized PB equation. In this case Eq. 19 can be written as

$$
\begin{aligned}
\Pi & =2 k_{B} \operatorname{Tn}_{0}\left(\cosh \beta e_{0} \phi(r=R)-1\right)= \\
& =2 k_{B} T n_{0}\left(\beta e_{0}\right)^{2} \phi^{2}(r=R)+\ldots=\kappa_{D}^{2} \epsilon \epsilon_{0} \phi^{2}(n=R)+\ldots,
\end{aligned}
$$

and represents the osmotic pressure difference between the wall of the cell and the bulk reservoir. Using now the solution of the linearized PB equation Eq. 13, we end up with the following expression for $\Pi$

$$
\Pi=\frac{\sigma^{2}}{\epsilon \epsilon_{0} K_{1}^{2}\left(\kappa_{D} a\right)} K_{0}^{2}\left(\kappa_{D} R\right) p^{2}\left(\kappa_{D} R, \kappa_{D} a\right),
$$

where we introduced the correction factor $p(x, y)$ as

$$
p\left(\kappa_{D} R, \kappa_{D} a\right)=\frac{1+\frac{K_{1}\left(\kappa_{D} R\right) I_{0}\left(\kappa_{D} R\right)}{I_{1}\left(\kappa_{D} R\right) K_{0}\left(\kappa_{D} R\right)}}{1-\frac{K_{1}\left(\kappa_{D} R\right) I_{1}\left(\kappa_{D} a\right)}{I_{1}\left(\kappa_{D} R\right) K_{1}\left(\kappa_{D} a\right)}} .
$$

The factor $p^{2}\left(\kappa_{D} R, \kappa_{D} a\right)$ obviously represents the effect of the finite concentration of the macromolecules, i.e. DNA, or in other words the effect of the walls of the cell. For small DNA densities this correction factor goes to unity, since in that limit only the first neighbors of the central molecule are important. This would be equivalent to taking only the first neighbors into account in the evaluation of the free energy. 
Eq. 24 for osmotic pressure is relatively complicated. In order to avoid the details that will later prove to be irrelevant, we investigate only its asymptotic form, valid for large values of $\kappa_{D} R$ or small values of the macromolecular concentration. In this limit we have $p\left(\kappa_{D} R, \kappa_{D} a\right) \longrightarrow 1$ and the approximate form of the osmotic pressure can be derived as

$$
\Pi \sim \frac{\sigma^{2}}{\epsilon \epsilon_{0} K_{1}^{2}\left(\kappa_{D} a\right)} K_{0}^{2}\left(\kappa_{D} R\right)=\frac{\sigma^{2}}{\epsilon \epsilon_{0} K_{1}^{2}\left(\kappa_{D} a\right)} \frac{\pi}{2} \frac{e^{-2 \kappa_{D} R}}{\kappa_{D} R},
$$

where we have taken into account the asymptotic form of the Bessel function $K_{0}(x) \longrightarrow$ $\sqrt{\frac{\pi}{2}} \exp (-x) x^{-1 / 2}$. From the expression for osmotic pressure Eq. 22 we can now derive the interaction free energy per unit length between the central molecule and its neighbors. We obtain

$$
\mathcal{F}(R) / L \sim \frac{\pi^{2}}{2} \frac{\sigma^{2}}{\epsilon \epsilon_{0} \kappa_{D}^{2} K_{1}^{2}\left(\kappa_{D} a\right)} e^{-2 \kappa_{D} R}=k_{B} T \frac{\ell_{B}}{b^{2}} e^{-2 \kappa_{D} R} .
$$

Here we introduced an effective separation $b$ between charges along the cylinder $1 / b=$ $\pi \sigma \lambda_{D} / \sqrt{2} e_{0} K_{1}\left(a / \lambda_{D}\right)$ [14] ( for DNA $b \sim l_{P O_{4}}$, where $l_{P O_{4}} \sim 1.7 \AA$, is the separation between the phosphate charges along DNA [15]). This expression derived via the cell model with cylindrical symmetry is very close to the expression derived via a pairinteraction energy evaluated on the linearized PB level [14]. This is not surprising since apart from geometric factors the cell model and the pairwise free energy should give the same result at vanishing macroion concentrations.

In the case of very large surface charges the nonlinearities of the Poisson-Boltzmann equation effectively change the surface charge density entering the above equation Eq. 27 via $b \longrightarrow \ell_{B}$ while leaving the separation dependence largely unaltered [6]. Sometimes these non-linearity effects, that become pronounced depending on whether the s.c. Manning parameter $\zeta={ }_{B} / b$ is larger or smaller than one, would be referred to as Manning condensation [13].

\section{EQUATION OF STATE: NO THERMAL FLUCTUA- TIONS}

An equation of state in general means a connection between the osmotic pressure $\Pi$ and the macromolecular density $n_{M}$ of an assembly

$$
\Pi=\Pi\left(n_{M}\right)
$$

and can in principle be obtained exactly via a complete statistical mechanical treatment of a solution composed of cylindrical macromolecules and the bathing medium. Since this is usually not feasible, a helpful shortcut is to evaluate the osmotic pressure in the cell model, that mimmicks the finite meacromolecular density as explained above.

The system that we are studying is composed of macomolecules that have intermolecular as well as intramolecular degrees of freedom since they are usually not infinitely rigid. A most naive approach to the equation of state would be to simply forget the intramolecular degrees of freedom, assume that the macromolecules are ideally rigid and 
that they assemble into a crystal of hexagonal symmetry with perfect positional order. In this case the osmotic pressure of such a system can be obtained via Ewald force summation (in the case of short range interactions even this is dispensable) involving the intermolecular potentials leading directly to the equation of state or again via the cell model; the latter approach being certanly simpler then the Ewald summation since it is effectively a single particle model. The osmotic pressure, i.e. the equation of state, in the cell model would be given by

$$
\Pi\left(n_{M}\right)=-\left(\frac{\partial(\mathcal{F}(R) / L)}{2 \pi R \partial R}\right)_{T} \mathcal{F}(R)=k_{B} T \frac{\ell_{B}}{b^{2}} e^{-2 \kappa_{D} R} .
$$

$L$ is the length of the molecules in the array. As already stated in writing this free energy we assumed that the van der Waals interactions as well as the short range, nonelectrostatic structural nteractions do not make an essential contribution to the equation of state. For the latter this is true only at not very high ionic concentrations and not too high concentration of the macromoleculs (for details see [19]).

We can now take this equation of state and compare it to experiments performed on DNA [19] at various solution conditions. After performing this type of exercise, one is immediately convinced that something crucial is missing as there is practically no correspondence (except at very high densities) between the experiment and this type of simpleminded theory, see Fig. 2. In the case of DNA the magnitude of the surface charge is taken at the Manning value [15], thus $b=\ell_{B}$. Obviously this equation of state underestimates the energetics of the system. In the following section we will try to ammend it by taking into consideration also the intramolecular degrees of fredom of the macromolecular ions.

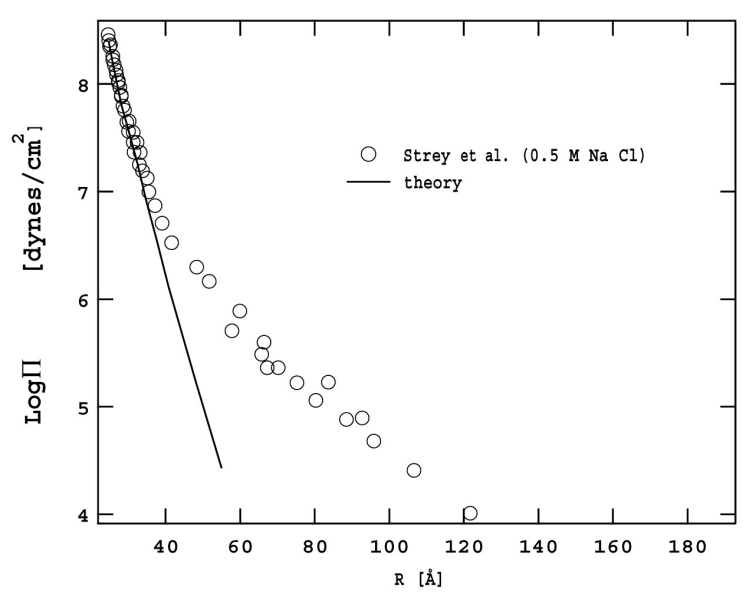

Figure 2. A comparison of the equation of state Eqs. 29 with DNA experimental data [19]. This form of the equation of state assumes that the molecules are infinitely rigid and crystalline. The magnitude of the electrostatic part of the interaction is obtained by assuming that the charge density of the DNA is correctly given by the Manning value [15]. Obviously there are large discrepancies on this level between the theoretical predictions and actual data. 


\section{EQUATION OF STATE: THE EFFECT OF THER- MAL FLUCTUATIONS (1)}

While the intermolecular degrees of freedom are taken into account through interaction potentials described in the previous section, the intramolecular degrees of freedom are usually treated within a mesoscopic elastic model that substitutes macroscopic elasticity for the complicated short-range intramolecular potentials acting between different segments of the macromolecules [17]. Elastic models for cylindrical macromolecules have been well worked out [18]. The general idea is that the trace over all the microscopic degrees of freedom is assumed to result exactly in the mesoscopic Hamiltonian itself, that one then uses in the partition function to evaluate the trace over mesoscopic degrees of freedom.

Because in the regime of relevant densities the hexagonal array is either in the line hexatic or the cholesteric phases [19] I can use the mesoscopic elastic Hamiltonians pertaining to them. The goal here will be to combine the effects of thermally driven elastic fluctuations of the macromolecules with the interactions between them and calculate their combined effect on the equation of state. This approach is based on ideas first introduced by Helfrich in the '70's and later worked out in detail by Lipowsky, Leibler and others in the '80's [20], who showed in the context of membranes that thermal conformational fluctuations can have a profound effect on interactions between flexible macromolecules.

\subsection{A macroscopic theory of the equation of state in an ordered macromolecular array}

Let us first consider the elastic free energy of a nematic: a three dimensional liquid with long-range orientational order with an average director $\mathbf{n}$ along the $\mathbf{z}$-axis. Such phases are typically formed by solutions of rod-like or disc-like objects. There are three kinds of deformations in quadratic order of $\mathbf{n}$ with symmetry $C_{\infty h}$ : splay, twist and bending. The corresponding elastic constants for these deformations are the Frank constants $K_{1}$, $K_{2}$ and $K_{3}[17]$.

$$
\mathcal{F}_{N}=\frac{1}{2} \int d^{2} \mathbf{r}_{\perp} d r_{z}\left[K_{1}(\nabla \cdot \mathbf{n})^{2}+K_{2}(\mathbf{n} \cdot(\nabla \times \mathbf{n}))^{2}+K_{3}(\mathbf{n} \times(\nabla \times \mathbf{n}))^{2}\right]
$$

For small deviations of the director field $\mathbf{n}(\mathbf{r})$ around its average orientation along the $z$ $\operatorname{axis} \mathbf{n}(\mathbf{r}) \approx\left(\delta n_{x}(\mathbf{r}), \delta n_{y}(\mathbf{r}), 1\right)$, the free energy assumes the form

$$
\mathcal{F}_{N}=\frac{1}{2} \int d^{2} \mathbf{r}_{\perp} d z\left[K_{1}\left(\nabla_{\perp} \cdot \delta \mathbf{n}\right)^{2}+K_{2}\left(\nabla_{\perp} \times \delta \mathbf{n}\right)^{2}+K_{3}\left(\partial_{z} \delta \mathbf{n}\right)^{2}\right],
$$

where $\boldsymbol{\nabla}=\left(\nabla_{\perp}, \partial_{z}\right)$. For polymer nematics we now have to consider that the director field and the density of polymers in the (x,y)-plane $\rho=\rho_{0}+\delta \rho$ are coupled [21, 22]. If the polymers were infinitely long and stiff the coupling is given by the continuity equation:

$$
\partial_{z} \delta \rho+\rho_{0} \nabla_{\perp} \cdot \delta \mathbf{n}=0
$$

This constraint, however, is softened if the polymer has a finite length $L$ or a finite persistence length $\mathcal{L}_{p}$. The persistence length is defined through the bending modulus of a 
single chain $K_{C}$ as $K_{C}=k_{B} T \mathcal{L}_{p}$. For DNA the persistence length is $\mathcal{L}_{p} \sim 50 \mathrm{~nm}$. The total bending elastic constant then has the form $K_{3}=\rho_{M} K_{C}$, where $\rho_{M}$ is the 2D density of the polymers perpendicular to their long axes.

On length scales larger than $\mathcal{L}_{p}$ the polymer can either fill the voids with its own ends or fold back on itself [23]. On these length scales the polymer nematic can splay without density change. Following [24] this can be expressed by introducing G, a measure of how effectively the constraint is enforced. Density changes are expanded to second order in density deviations $\delta \rho\left(\mathbf{r}_{\perp}, z\right)=\rho\left(\mathbf{r}_{\perp}, z\right)-\rho_{0} . \mathcal{B}$ is the bulk modulus for compressions and dilations normal to the chains. The total free energy can now be written as

$$
\mathcal{F}=\mathcal{F}_{0}\left(\rho_{0}\right)+\frac{1}{2} \int d^{2} \mathbf{r}_{\perp} d z\left[\mathcal{B}\left(\frac{\delta \rho}{\rho_{0}}\right)^{2}+G\left(\partial_{z} \delta \rho+\rho_{0} \nabla_{\perp} \cdot \delta \mathbf{n}\right)^{2}\right]+\mathcal{F}_{N}
$$

where $\mathrm{G}$ is given by $G=\frac{k_{B} T L}{2 \rho_{0}}$ where $L$ can not exceed $\mathcal{L}_{p}$ [24]. In the limit of finite polymer length $\mathrm{G}$ is also finite and can be obtained from the observation that $\partial_{z} \delta \rho+\rho_{0} \nabla_{\perp} \cdot \delta \mathbf{n}$ equals the difference between the number of polymer heads and tails [22]. From here one derives that $G$ is the concentration susceptibility for an ideal mixture of heads and tails thus $G=k_{B} T /\left(\rho_{H}+\rho_{T}\right)$, where $\rho_{H}$ and $\rho_{T}$ are the average concentrations of heads and tails, with $\rho_{H}, \rho_{T}=\rho_{M}$. The macromolecular density on the other hand equals $\rho_{M}=\rho_{0} / L$, wherefrom $G=k_{B} T \ell / 2 \rho_{0}$. The corresponding structure factor can be written as

$$
\mathcal{S}\left(q_{\perp}, q_{z}\right)=\left\langle\left|\delta \rho\left(q_{\perp}, q_{z}\right)\right|^{2}>=k_{B} T \frac{\rho_{0}^{2} q_{\perp}^{2}+k_{B} T \frac{\mathcal{K}(\mathbf{q})}{\mathcal{G}}}{\mathcal{B} q_{\perp}^{2}+k_{B} T\left(\frac{\mathcal{B}}{G \rho_{0}^{2}}+q_{z}^{2}\right) \mathcal{K}(\mathbf{q})}\right.
$$

where we defined

$$
\mathcal{K}(\mathbf{q})=\frac{K_{1} q_{\perp}^{2}+K_{3} q_{z}^{2}}{k_{B} T} .
$$

For long-fragment DNA the limit $L \longrightarrow \infty$ is appropriate, leading to the structure factor proposed by Selinger and Bruinsma [25]

$$
\mathcal{S}\left(q_{\perp}, q_{z}\right)=k_{B} T \frac{\rho_{0}^{2} q_{\perp}^{2}}{K_{1} q_{\perp}^{2} q_{z}^{2}+K_{3} q_{z}^{4}+\mathcal{B} q_{\perp}^{2}} .
$$

In order to calculate the contribution to the free energy due to fluctuations in nematic order we have to sum over all the density modes, obtaining

$$
\mathcal{F}=\frac{1}{2} k_{B} T \iint \frac{d^{2} q_{\perp} d q_{z}}{(2 \pi)^{3}} \log \left(K_{1} q_{\perp}^{2} q_{z}^{2}+K_{3} q_{z}^{4}+\mathcal{B} q_{\perp}^{2}\right) .
$$

The problem here is that the above integral requires a cutoff and that the higher order terms in $q_{\perp}$ are more important than the ones we have kept here. For a moment let us assume that this free energy is valid and we may calculate

$$
\frac{\partial \mathcal{F}}{\partial \mathcal{B}}=\frac{1}{2} k_{B} T V \iint \frac{q_{\perp} d q_{\perp} d q_{z}}{(2 \pi)^{2}} \frac{q_{\perp}^{2}}{K_{1} q_{\perp}^{2} q_{z}^{2}+K_{3} q_{z}^{4}+\mathcal{B} q_{\perp}^{2}} .
$$

The $q_{z}$ integral can be done straightforwardly and we remain with

$$
\frac{\partial \mathcal{F}}{\partial \mathcal{B}}=\frac{1}{2} k_{B} T \frac{V}{(2 \pi)^{2}} \frac{\pi}{2} \int \frac{q_{\perp}^{3} d q_{\perp}}{\sqrt{\mathcal{B} q_{\perp}^{2}} \sqrt{K_{1} q_{\perp}^{2}+2 \sqrt{\mathcal{B} K_{3} q_{\perp}^{2}}}} .
$$


This integral depends essentially on the upper cutoff for $q_{\perp}=q_{\perp \max }$ and we obtain

$$
\frac{\partial \mathcal{F}}{\partial \mathcal{B}}=k_{B} T \frac{V}{4 \pi} \frac{\mathcal{B} K_{3}}{K_{1}^{2} \sqrt{\mathcal{B} K_{1}}} F\left(\frac{q \perp \max }{2 \sqrt{\frac{\mathcal{B} K_{3}}{K_{1}^{2}}}}\right),
$$

where the function $F(x)$ has been defined as

$F(x)=\int_{0}^{x} \frac{u^{3 / 2} d u}{\sqrt{1+u}}=\frac{1}{4}(\sqrt{x} \sqrt{1+x}(2 x-3)+3 \operatorname{areasinh} \sqrt{x})=\left\{\begin{array}{ll}\frac{2}{5} x^{5 / 2} & ; x \ll 1 \\ \frac{1}{2} x^{2} & ; x \gg 1\end{array}\right.$.

From here we obtain the two limiting forms of the free energy as

$$
\begin{aligned}
\mathcal{F} & \simeq \frac{k_{B} T V}{5 \times 2^{3 / 2} \pi} \sqrt[4]{\frac{\mathcal{B}}{K_{3}}} q_{\perp \text { max }}^{5 / 2}+\ldots \quad ; \quad q_{\perp \text { max }} \ll 2 \sqrt{\frac{\mathcal{B} K_{3}}{K_{1}^{2}}} \\
\mathcal{F} & \simeq \frac{k_{B} T V}{16 \pi} \sqrt{\frac{\mathcal{B}}{K_{1}}} q_{\perp \text { max }}^{2}+\ldots \quad ; \quad q_{\perp \text { max }} \gg 2 \sqrt{\frac{\mathcal{B} K_{3}}{K_{1}^{2}}} .
\end{aligned}
$$

Obviously the long-wavelength physics is very complicated and depends crucially on the values of typical polymer length and the ratios of elastic constants. However it is also dependent on the $q_{\perp}$ cutoff. We have to either eliminate the cutoff by including higher order terms in the original Hamiltonian or choose a meaningful cutoff. Higher order terms will capture the short-wavelength physics and remove the divergence [19].

One can show [19] that a consistent value of the cutoff has to be proportional to the Brillouin zone radius $q_{\perp \max } \simeq \frac{\pi}{D}$, where $D$ is the effective separation between the polymers in the nematic phase. This is a physically meaningful and appropriate cutoff because the underlying macroscopic elastic model has, by definition, to break down at wavelengths comparable to the distance between molecules.

Putting in the numbers valid for DNA arrays one realizes that in the regime of densities considered here we are always in the Eq. 42 limit. We would now have to derive the mesoscopic elastic moduli from the microscopic interactions described via a pair potential $\mathcal{F}(R)$ (the interaction free energy) between the segments of the macromolecules. At present this program is too ambitious and we simply exploit the standard ansatz for the different elastic moduli [21] expressed via the cell model free energy

$$
\begin{aligned}
K_{1} & =K_{2} \simeq \mathcal{F}(R) / R \\
K_{3} & \simeq \rho_{0} K_{C}+\mathcal{F}(R) / R \\
\mathcal{B} & \simeq V \frac{\partial^{2} \mathcal{F}(V)}{\partial V^{2}}=\frac{1}{4 \pi}\left(\frac{\partial^{2}(\mathcal{F} / L)}{\partial^{2} R}-\frac{1}{R} \frac{\partial(\mathcal{F} / L)}{\partial R} .\right),
\end{aligned}
$$

where $\rho_{0}$ is the $2 \mathrm{D}$ density of the macromolecules perpendicular to their long axes, $K_{C}$ is the elastic rigidity modulus of a single polymer molecule and we assumed that the polymers have an average separation $R$ between first neighbors [19].

The macroscopic free energy Eq. 42 together with the values of elastic constants Eq. 44 already points to the salient features of the fluctuation modified equation of state. Obviously the thermal fluctuations make the free energy much longer ranged than the 
underlying microscopic interaction potential. If the interaction potential decays exponentially with characteristic length $\lambda$, then the free energy Eq. 42 decays with four times the characteristic length! The factor of four is a simple consequence of mesoscopic elasticity.

We now use the form of the bare interaction free energy $\mathcal{F}(R)$ appropriate for a DNA array Eq. 29. One can see that at all relevant densities $K_{3} \simeq \rho_{0} K_{C}$. We are now able to fit the calculated equation of state obtained from Eq. 42 to the experimental equation of state [19], see Fig. 3. The values for the DNA bending rigidity and the Debye length obtained from such a fit are comfortably within the expected range [19]. Let me just mention here that the effective charge evaluated from the fit is about half the amount expected on the basis of the Manning condensation theory. A fundamental drawback

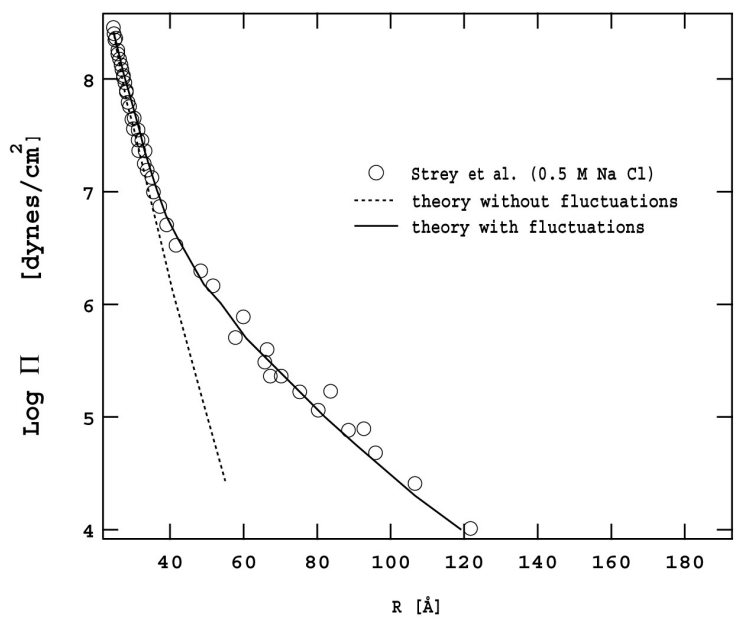

Figure 3. A fit of the lowest order fluctuation equations of state, Eqs. 42, to DNA [19] data. Dashed line - theory without fluctuations as on FIg. 2. Full line - theory with conformational fluctuations of the molecules taken into accoumt on a harmonic level, 42. The value of the effective charge on the DNA surface is obtained from the fit to experiment and is found to be about half the Manning condensation value.

of this formulation for the equation of state in an assembly of flexible molecules is most clearly seen in the ansatz Eq. 44 . The elastic moduli are not really calculated on the same level as the free energy but are assumed to have a form, that at least for the compressibility modulus, would be strictly valid only for rigid molecules. The above formulation is thus not completely self-consistent and we will make an attempt to improve it in the next section. The failure of this attempt will make us aware of some fundamental properties of the nature of the positional order in DNA arrays. 


\section{EQUATION OF STATE: THE EFFECT OF THER- MAL FLUCTUATIONS (2)}

At this point one can use any of the advanced theories that take into account the thermal fluctuations at a deeper level than the macroscopic theory of the previous section and that allow also for the thermal fluctuation effects that can modify the compressibility modulus itself [26]. Such theories are well worked out also in other areas of physics such as e.g. magnetic vortex arrays in type II superconductors. There are different approaches that one can follow. One could either formulate the problem in the language of the functional renormalization group [26] or on the level of a variational calculation of the compressibility modulus in the Feynman - Kleinert style [27]. To remain as close as possible to the approach outlined before, we chose the second, i.e. variational, approach. It usually fares quite well even when compared to the more powerful renormalization group approach.

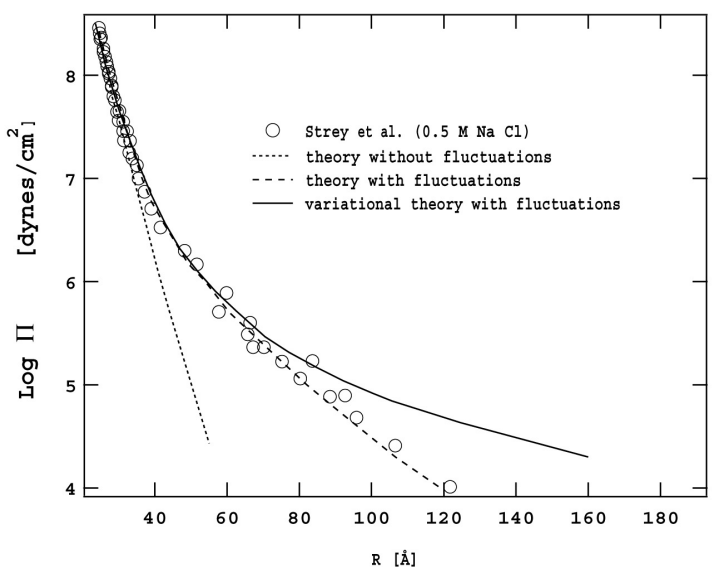

Figure 4. A fit of the variational equation of state to the experimental data. Here the values of the elastic moduli are obtained self consistently and also include a fluctuation contribution. Surprisingly this fit with a variational equation of state for DNA obtained from Eq. 54 fares much worse than the macroscopic fluctuation theory, see Fig. 3. The reason lies in the nature and range of the positional order in an ordered assembly of DNA, see main text.

\subsection{Variational calculation of the osmotic pressure in a hexagonal array}

For a system with a hexagonal local symmetry I follow closely the calculation of Volmer and Schwartz [28] derived for the system of magnetic vortex lines in type II superconductors. Apart from the difference between elastic energies of a vortex line vs. a flexible polymer the two cases are analogous. The interaction Hamiltonian of an oriented DNA 
polymer array with hexagonal local symmetry can be written in the form

$$
\mathcal{H}=\frac{1}{2} K_{C} \sum_{n, m=1}^{\infty} \int d z\left(\frac{\partial^{2} \mathbf{r}_{\perp}{ }^{(n, m)}(z)}{\partial z^{2}}\right)^{2}+\frac{1}{2} \sum_{n, m \neq n^{\prime} m^{\prime}}^{\infty} \int d z V\left(\mathbf{r}_{\perp}{ }^{(n, m)}(z)-\mathbf{r}_{\perp}{ }^{\left(n^{\prime}, m^{\prime}\right)}(z)\right)
$$

where $\mathbf{r}_{\perp}{ }^{(n, m)}(z)$ is the local displacement of a polymer chain at the $(n, m)$ lattice position perpendicular to the long axis, $z$, while $V\left(\mathbf{r}_{\perp}{ }^{(n, m)}(z)-\mathbf{r}_{\perp}{ }^{\left(n^{\prime}, m^{\prime}\right)}(z)\right)$ is the interaction potential between different macromolecules at the same value of $z$. In principle the indices $n, m$ would run through all the positions of the polymers at a certain planar cross section through the nematic but because of the short range nature of the interaction and computational convenience I restrict them to nearest neighbors [28]. $K_{C}$ is of course the elastic modulus of DNA given by $K_{C}=k_{B} T \mathcal{L}_{P}$, where $\mathcal{L}_{P}$ is the persistence length.

Instead of using this nonharmonic Hamiltonian I will take a simpler reference Hamiltonian of a general harmonic form. Let us start with the following parametrization

$$
\begin{aligned}
\mathbf{r}_{\perp}{ }^{(n, m)}(z) & =\mathbf{R}_{n m}+\mathbf{u}^{(n, m)}(z) \\
\mathbf{u}^{(n, m)}(z) & =\sum_{\mathbf{Q}_{\perp}, q_{z}} \mathbf{u}\left(\mathbf{Q}_{\perp}, q_{z}\right) e^{i q_{z} z+i \mathbf{Q}_{\perp} \mathbf{R}_{n m}}
\end{aligned}
$$

where $\mathbf{R}_{n m}=n \mathbf{a}_{1}+m \mathbf{a}_{2}$, with $\mathbf{a}_{1}$ and $\mathbf{a}_{2}$ the two basis vectors of the macomolecular lattice perpendicular to the long axis $z$ of the molecules and $\mathbf{Q}_{\perp}$ the appropriate reciprocal lattice vectors. I take the reference Hamiltonian in the general harmonic form in the reciprocal Fourier space

$$
\mathcal{H}_{0}=\frac{1}{2} \sum_{\mathbf{Q}_{\perp}, q_{z}}\left(K_{C} q_{z}^{4} \delta_{i k}+\mathcal{B}_{i k}\left(\mathbf{Q}_{\perp}\right)\right) u_{i}\left(\mathbf{Q}_{\perp}, q_{z}\right) u_{k}\left(-\mathbf{Q}_{\perp},-q_{z}\right)+V_{0}(\mathbf{a}),
$$

where $\mathcal{B}_{i k}\left(\mathbf{Q}_{\perp}\right)=\mathcal{B}_{i k} \sum_{\mathbf{a}} 4 \sin ^{2} \frac{\mathbf{Q}_{\perp} \mathbf{a}}{2}$ and the sum over a refers to summation over the positions of nearest neighbors.

The idea of the Feynman - Kleinert variational principle is now to use the Hamiltonian Eq. 47 as an harmonic ansatz whose effective parameters, like $\mathcal{B}_{i k}$ and $V_{0}(\mathbf{a})$ are determined variationally by minimizing the upper bound for the free energy [27] . This approach has already been used in the context of multilamellar systems [29].

Let us start with what is usually referred to as the Gibbs - Bogolyubov inequality. Taking the exact free energy, corresponding to the Hamiltonian $\mathcal{H}$ and an approximate one, corresponding to $\mathcal{H}_{0}$, it is straightforward to derive

$$
\mathcal{F} \leq \mathcal{F}_{0}+\left\langle\mathcal{H}-\mathcal{H}_{0}\right\rangle_{\mathcal{H}_{0}}
$$

The average $\langle\ldots\rangle_{\mathcal{H}_{0}}$ is performed with respect to the Hamiltonian Eq. 47. Obviously the above inequality defines an upper bound for the free energy. By evaluating explicitly the terms in Eq. 48 with the reference Hamiltonian given by Eq. 47, one is left with

$$
\mathcal{F}_{0}=\frac{1}{2} \sum_{\mathbf{Q}_{\perp}, q_{z}}\left(K_{C} q_{z}^{4} \delta_{i k}+\mathcal{B}_{i k}\left(\mathbf{Q}_{\perp}\right)\right)\left\langle u_{i}\left(\mathbf{Q}_{\perp}, q_{z}\right) u_{k}\left(-\mathbf{Q}_{\perp},-q_{z}\right)\right\rangle_{\mathcal{H}_{0}}+V_{0}(\mathbf{a}),
$$


where the positional correlation function is given by

$$
\left\langle u_{i}\left(\mathbf{Q}_{\perp}, q_{z}\right) u_{k}\left(-\mathbf{Q}_{\perp},-q_{z}\right)\right\rangle_{\mathcal{H}_{0}}=\sigma_{i k}\left(\mathbf{Q}_{\perp}, q_{z}\right)=\frac{k_{B} T}{K_{C} q_{z}^{4} \delta_{i k}+\mathcal{B}_{i k}\left(\mathbf{Q}_{\perp}\right)},
$$

via the equipartition theorem since we have a general quadratic form of the reference Hamiltonian. On the other hand the second term of Eq. 48 can be derived as

$$
\left\langle\mathcal{H}-\mathcal{H}_{0}\right\rangle_{\mathcal{H}_{0}}=\frac{1}{2} V_{\sigma_{i j}\left(\mathbf{Q}_{\perp}, q_{z}\right)}(\mathbf{a})-2 \mathcal{B}_{i j} \sum_{\mathbf{Q}_{\perp}, q_{z}} \sum_{\mathbf{a}} \sin ^{2} \frac{\mathbf{Q}_{\perp} \mathbf{a}}{2} \sigma_{i k}\left(\mathbf{Q}_{\perp}, q_{z}\right)-V_{0}(\mathbf{a}) .
$$

where in the first term of the above expression we introduced the fluctuation modified form of the interaction potential given by

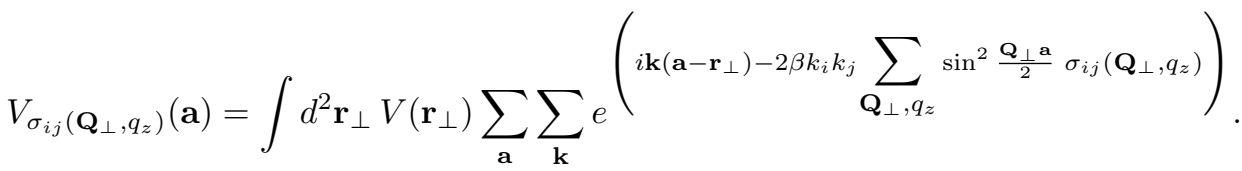

This simply follows from the fact that $\mathcal{H}_{0}$ is a quadratic function for which one has $\left\langle e^{i A_{i} u_{i}}\right\rangle_{\mathcal{H}_{0}}=e^{-\frac{1}{4} A_{i} A_{k}\left\langle u_{i} u_{k}\right\rangle_{\mathcal{H}_{0}}}$. All this is very closely related the analysis of Volmer and Schwartz [28] for the system of magnetic vortex lines in type II superconductors. The only difference is in the conformational energy of a polymer (elastic energy) and a vortex line (tension energy). The above variational formulation is obviously based on two parameters: $\sigma_{i k}\left(\mathbf{Q}_{\perp}, q_{z}\right)$ and $V_{0}(\mathbf{a})$.

What the Feynman - Kleinert variational principle is aiming at is to minimize the second term of Eq. 48. Let us first consider the minimal value of $V_{0}(\mathbf{a})$. Clearly the second term of Eq. 48 is non-negative. It is in fact minimal if

$$
V_{0}(\mathbf{a})=\frac{1}{2} V_{\sigma_{i j}\left(\mathbf{Q}_{\perp}, q_{z}\right)}(\mathbf{a})-\frac{1}{2} \sum_{\mathbf{Q}_{\perp}, q_{z}} \mathcal{B}_{i k}\left(\mathbf{Q}_{\perp}\right) \sigma_{i j}\left(\mathbf{Q}_{\perp}, q_{z}\right),
$$

making thus $\mathcal{F}=\mathcal{F}_{0}$ or in extenso

$$
\mathcal{F}_{0}=-k_{B} T \log \left\langle e^{-\beta \mathcal{H}_{0}}\right\rangle=V_{0}(\mathbf{a})+\frac{k_{B} T}{2} \operatorname{Tr} \sum_{\mathbf{Q}_{\perp}, q_{z}} \log \left(\delta_{i k}+\frac{\mathcal{B}_{i k}\left(\mathbf{Q}_{\perp}\right)}{K_{C} q_{z}^{4}}\right) .
$$

It is obvious from the above expression what the fluctuations do to the free energy on this level of approximations. Without the thermal noise the second term of Eq. 54 would be zero, and we would be back to the equation of state without any intramolecular degrees of freedom. The fluctuations again, just as in the previous section, effectively boost the bare intermolecular interactions this time quantified by $V_{0}(\mathbf{a})$.

As for the second parameter that needs to be minimized, one gets simply

$$
2 \mathcal{B}_{i j} \sum_{\mathbf{a}} \sin ^{2} \frac{\mathbf{Q}_{\perp} \mathbf{a}}{2}=\frac{1}{2} \frac{\partial V_{\sigma_{i j}\left(\mathbf{Q}_{\perp}, q_{z}\right)}(\mathbf{a})}{\partial \sigma_{i j}\left(\mathbf{Q}_{\perp}, q_{z}\right)} .
$$

Equations Eq. 53 and 55 represent the solution to the minimization problem. Very similar equations have already been derived in the case of magnetic vortex arrays [28]. 
The most important quantity of the above formulation of the fluctuational renormalization of the interactions in a macromolecular array is the pair interaction potential per unit length between two polymer segments $V\left(\mathbf{r}_{\perp}{ }^{(n, m)}(z)-\mathbf{r}_{\perp}{ }^{\left(n^{\prime}, m^{\prime}\right)}(z)\right)$ given again by Eq. 29, that enters Eq. 54. One should appreciate the main difference between the calculation described in this section and the previous section: before the elastic moduli were given by different expressions, involving only the bare interaction Eq. 44; now the elastic modulus is given as a function of fluctuation renormalized interaction, Eq. 55. The last calculation should thus in principle be more accurate in describing the effect of thermal fluctuations.

However, when we compare the equation of state obtained from Eq. 54 with the macroscopic fluctuation equation of state from Eq. 42, we see that it fares much worse when compared with experiments, see Fig. 4. This fact is surprising, since the whole idea was to get an even better estimate of the fluctuation effects, and demands an explanation.

The reason for this discrepancy is found out to be quite simple. The variational ansatz based on positional correlation function $\sigma_{i k}\left(\mathbf{Q}_{\perp}, q_{z}\right)$ only makes sense if this quantity itself is well defined, thus if $\sigma_{i k}\left(\mathbf{Q}_{\perp}, q_{z}\right)<\infty$. This constraint fits best the description of a solid, with long range positional order, and finite correlations between any two macromolecular positions. DNA however, at the relevant densities, is not a solid. It is a hexatic liquid crystal with only short range positional order and is thus more akin to a fluid. For a fluid of course, with only short range positional correlations, the positional correlations (as opposed to density correlations which of course remain finite) diverge in the thermodynamic limit and can not be described with a finite correlation function. Therefore the variational theory, strictly applicable only to a hexagonal crystal, fares much worse than the mesoscopic fluctuation theory of the previous section, if applied to a DNA array at densities between the crystalline and isotropic phases.

A variational theory similar in spirit to what I developed above was also put forth by de Vries [30] basing his analysis on previous work by Odijk [31]. Numerical results of this approach are indistinguishable from those presented above.

\section{CONCLUSION}

I have given a broad overview of the work on the equation of state of DNA in aqueous ionic solutions. Most of what I described applies only to monovalent salt solutions. Higher valency salts have a very different effect on the properties of DNA [15] falling outside of my immediate interests. The picture that I developed here shows that depending on the monovalent salt concentration, conformational fluctuations mask the direct electrostatic interactions at all but very low salt. It might thus come as a surprise that for DNA, which is a very highly charged polyelectrolyte, the effect of electrostatic interactions on the equation of state is modified in an essentail way by the conformational fluctuations of DNAs. Direct electrostatic effects for this highly charged polyelectrolyte are thus counterintuitively discernible only in a very limited range of salt concentrations. 


\section{Acknowledgements}

I would like to thank Adrian Parsegian, Per Lyngs Hansen, Stephanie Tristram-Nagle and John Nagle for numerous discussions on the various aspects of the work described above. 


\section{Bibliography}

[1] R. Podgornik, H.H. Strey and V.A. Parsegian, Curr. Op. in Colloid and Interf. Sci. 3 (1998) 534.

[2] H.H. Strey, R. Podgornik, D.C. Rau and V.A. Parsegian, Curr. Opin. Struc. Biol. 8 (1998) 309-313.

[3] J. Israelachvili, Intermolecular and Surface Forces Hardcover 2nd edition Academic Press (1998)

[4] E.G. Verwey and J.T.G. Overbeek, The Theory of the Stability of Lyophilic Colloids (Elsevier, Amsterdam, 1948)

[5] J. Mahanty and B.W. Ninham, Dispersion Forces (Academic Press, London, 1976)

[6] D. Andelman, in Structure and Dynamics of Membranes, Eds. R. Lipowsky and E. Sackmann, North Holland, New York (1995) 603.

[7] R. Kjellander, in 'Electrostatis effects in soft matter and biophysics', C. Holm, P. Kekicheff and R. Podgornik Eds., NATO Science Series II - Mathematics, Physics and Chemistry, volume 46 (2001)

[8] R. Fuoss, A. Katchalsky, and S. Lifson, Proc. Natl. Acad. Sci USA 37 (1951) 579; S.Lifson, and Katchalsky A., J. Polymer Sci. XIII (1954) 43.

[9] M.N. Tamashiro, H. Schiessel H, Phys Rev E 68 (2003) art. no. 066106.

[10] I.A. Shkel, O.V. Tsodikov and M.T. Record Jr., J. Phys. Chem. 104 (2000) 51615170. I.A. Shkel, O.V. Tsodikov and M.T. Record Jr., Proc. Natl. Acad. Sci. 99 (2002) 2597-2602.

[11] Tracy CA, Widom H, Physica A 244 (1997) 402-413.

[12] W.M. Gelbart, R.F. Bruinsma, P.A. Pincus and V.A. Parsegian, Physics Today 53 (2000) 38 .

[13] K.S. Schmitz, Macroions in Solution and Colloidal Suspension, VCH Publishers, New York (1993).

[14] S. L. Brenner and V. A. Parsegian, Biophys. J. 14, 327-334 (1974).

[15] V.A. Bloomfield, D.M. Crothers and I. Tinoco, Nucleic Acids: structures, properties and functions, University Science Book, Sausalito (2000).

[16] B.V. Derjaguin, N.V. Churaev and V.M. Muller and, Surface Forces, Consultants Bureau, New York (1987). 
[17] P. M. Chaikin, T. C. Lubensky, Principles of Condensed Matter Physics (Cambridge Univ. Pr., 2000)

[18] A.G.. Petrov, The Lyotropic State of Matter: Molecular Physics and Living Matter Physics (Gordon and Breach Science Publishers, 1999).

[19] H.H. Strey, V.A. Parsegian and R. Podgornik, Phys. Rev. E 59 (1999) 999-1008. .

[20] R. Lipowsky, in Structure and Dynamics of Membranes, Eds. R. Lipowsky and E. Sackmann, North Holland, New York (1995) 521.

[21] P. De Gennes and J. Prost, The Physics of Liquid Crystals, 2nd ed. (Oxford University Press, Oxford, 1993).

[22] R. Meyer, in Polymer Liquid Crystals, edited by A. Ciferri, W. Krigbaum, and R. Meyer (Academic, New York, 1982), p. 133.

[23] A. Semenov and A. Khokhlov, Sov. Phys. Usp. 31, 988 (1988).

[24] D.R. Nelson, Defects and Geometry in Condensed Matter Physics, (Cambridge University Press, Oxford, 2002). March 2002 | Paperback (Hardback) | 392 pages 137 line diagrams 3 half-tones 2 tables | ISBN: 0521004004

[25] J. Selinger and R. Bruinsma, Phys. Rev. A 43, 2910 (1991).

[26] R. Lipowsky, in Structure and Dynamics of Membranes, Eds. R. Lipowsky and E. Sackmann, North Holland, New York (1995) 603.

[27] H. Kleinert, Path Integrals in Quantum Mechanics, Statistics, and Polymer Physics (World Scientific Pub Co, 1995)

[28] A. Volmer, M. Schwartz, Eur. Phys. J B 7211 (1999)

[29] R. Podgornik and V.A. Parsegian, Langmuir 8557 (1992)

[30] R. de Vries, J. Phys. II France 41541 (1994)

[31] T. Odijk, Langmuir 81690 (1992). T. Odijk, Europhys. Lett. 24177 (1993) . 